\title{
A Neuro-Fuzzy Model of Evaporator in Organic Rankine Cycle
}

\author{
Hamid Enayatollahi, Peter Fussey, Bao Kha Nguyen \\ Department of Engineering and Design, University of Sussex \\ Brighton BN1 9QT, UK \\ H.Enayatollahi@sussex.ac.uk; P.M.Fussey@sussex.ac.uk; B.K.Nguyen@sussex.ac.uk
}

\begin{abstract}
The Organic Rankine Cycle (ORC) is a propitious waste heat recovery (WHR) technology that allows recovery of wasted energy from low to medium temperature sources. This WHR method needs to be adopted as an Internal Combustion Engine (ICE) bottoming technology to mitigate its environmental effects and fulfil exhaust gas emission regulations. The evaporator is the most decisive element of the ORC cycle due to its high nonlinear behaviour and high thermal inertia. In this study, a neuro -fuzzy model of the evaporator is presented based on the data obtained from Finite Volume (FV) model of the evaporator. The simulation results are compared in terms of RMSE, error mean and standard deviation. The data obtained from ANFIS model reached a promising agreement with FV model. For prediction of the evaporator outlet temperature, RMSEs of 0.152 and 1.33 obtained for the training and test data, respectively. Furthermore, the ANFIS model was successfully able to predict the evaporator power with RMSE of 0.035 for the training and 0.2 for the test data. In addition, the ANFIS model compared to the FV model with twenty control volumes enhanced the simu lation time significantly. This clearly indicates the great potential of employing ANFIS model for real-time applications.
\end{abstract}

Keywords: Organic Rankine Cycle, Waste Heat Recovery, Evaporator Modelling, ANFIS, Neuro-Fuzzy Modelling.

\section{Introduction}

In the recent years, an increase in the greenhouse gas emissions has yielded to several drastic environmental footprints such as rises in the earth temperature and sea levels, the desertification, negative biodiversity consequences and so on. Most of these greenhouse gas emissions are released to the atmosphere by energy sector of industrialized countries in form of $\mathrm{CO}_{2}$, and development of other less-industrialized countries can speed up this process. Internal Combustion Engines (ICEs) are among the biggest producers of $\mathrm{CO}_{2}$ emissions due to their broad applications in transportation, and their low energy conversion efficiencies. An enhancement in efficiency of ICE's can help to reduce the production of greenhouse gases, thereby slowing down the process of earth global warming. Almost 70\% of the fuel energy in ICEs is wasted in form of the thermal energy from exhaust $(22-46 \%)$ and coolant $(18-42 \%)[1,2]$. In this regard, developing technologies to capture this wasted thermal energy without increasing the fuel consumption of ICEs and converting it into useful mechanical or electrical energy in vehicles can reduce harmful environmental effects of them.

The Organic Rankine Cycle (ORC) is one of the auspicious engine bottoming technologies, which is comprised of several components including evaporator, expander, condenser and pump [3, 4]. As shown in Fig. 1, the working principle of the ORC is like Steam Rankine Cycle (SRC) whereas instead of using the water-steam power as a means of propulsion, an organic medium which features higher molecular weight and lower boiling temperature is evaporated as the working fluid to provide the required electromotive force [5]. The higher molecular weight of organic fluid compared to the water in ORC, reduces the required pumping work in the cycle and thus, enhances the efficiency of cycle [5]. Adopting the ORC for mobile applications is a challenging task. Unlike the steady profile heat sources, the temperature and mass flow rate of exhaust gases in ICEs change dramatically during the driving cycles [2]. This transient heat source has a direct influence on the evaporating power of the cycle. To ensure the safe operation of the ORC and increase its efficiencies, accurate and reliable control strategies should be developed [6]. Furthermore, accuracy of control strategies is dependent on the precise modelling of all the components within the ORC cycle. The evaporator is known as the most critical component of the ORC cycle because of high nonlinearity of its governing equations and its high thermal inertia. Other components of the ORC cycle have much faster response time compared to the evaporator. In other words, because of high thermal inertia, the evaporator behaviour dominates the dynamic response of the ORC. Therefore, creating a dynamic model of the evaporator that is capable of handling transient conditions in an ORC waste heat recovery system is inevitable. 


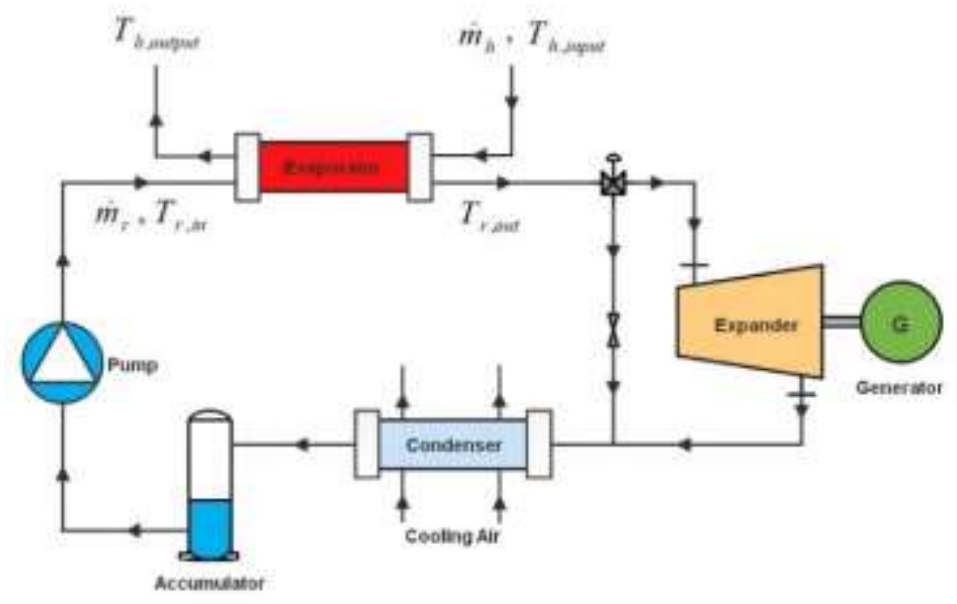

Fig. 1: Conceptual schematic of ORC system.

Two main dynamic physic-based models of evaporator can be found in the literature: Finite Volume (FV) and Moving Boundary (MB). As it is depicted in Fig. 2, FV method discretizes the evaporator length to a finite number of control volumes and deploys the numerical methods to solve the heat transfer equations between the hot fluid, the evaporator's wall and the working fluid [7]. Number of the control volumes has an influence on the accuracy and the computational time of the model. Choosing higher number of control volumes will result in a model, which is computationally more expensive. In contrast, MB method is a control-oriented approach that discretizes the length of evaporator to three control volumes with timevarying intermediate boundaries. Reducing the number of control volumes causes fewer states in the equations of MB method compared to FV method [6], but despite fewer states, this method is still computationally expensive. Moreover, nonexistence of any of the distinct fluid phases will result in singularities in the equations of MB method.

Logic-based methods of the evaporator modelling such as fuzzy method has been implemented recently to model the evaporator behaviour $[7,8]$. This method can reduce the computational time of the model substantially owing to the absence of time-consuming calculations of heat transfer equations. Input-output mapping of fuzzy system is obtained by setting the rules and membership functions for input and output of the system, which requires incorporation of expert's knowledge about the system behaviour. Despite faster speed of fuzzy method compared to the physic-based methods, because of the complex behaviour of evaporator nature, tuning the rules and membership functions of fuzzy system is a time-consuming task. In addition, with a slight change in the evaporator's geometrical layout the accuracy of model drops dramatically, and the fuzzy rules and membership functions need to be adjusted accordingly. Therefore, the aim of this study is to adopt the ANFIS method, which utilizes pattern recognition power of neural networks in conjunction with modelling benefits of fuzzy systems to reduce the development time of fuzzy model of the evaporator and improve its accuracy.

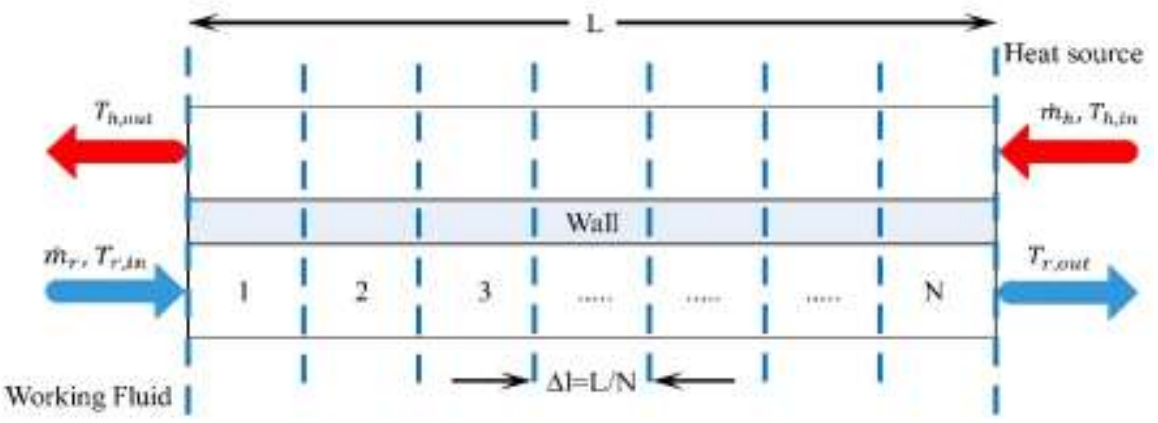

Fig. 2: Discretization of evaporator to N control volumes. 


\section{Adaptive Network-Based Fuzzy Inference System (ANFIS)}

ANFIS is an artificial intelligence method introduced in 1993 by Jang [9]. This technique maps the input data to the output data by combining feedforward neural networks and Takagi-Sugeno (TSK) fuzzy inference system (FIS). Utilizing pattern recognition power of neural networks in the structure of ANFIS method eliminates the dependency of fuzzy system design on expert's knowledge about the system behaviour and improves the accuracy of its prediction by setting the optimal location of membership functions in the fuzzy sets. Fig. 3 represents a typical architecture of ANFIS for a system with two inputs and one output. The ANFIS architecture has five layers and is formed by representing the TSK fuzzy inference system in the framework of feedforward neural network. Each layer of ANFIS architecture is comprised of several nodes. These nodes can be either fixed nodes accomplishing a certain task like addition and multiplication (layers 2, 3 and 5), or adaptive nodes with tuneable parameters (Layers 1 and 4). By interconnection of the layers, the ANFIS network is formed.

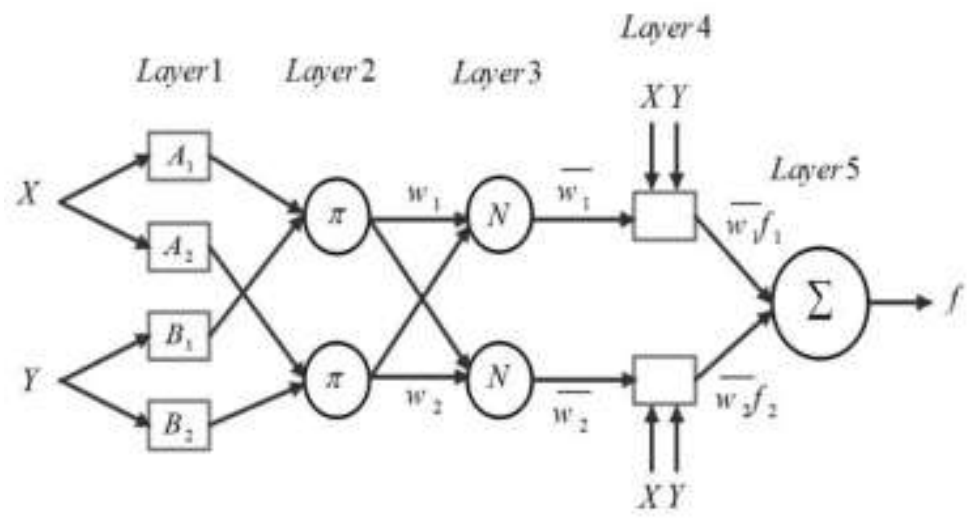

Fig. 3: ANFIS architecture.

To illustrate the rule base of the ANFIS system with two inputs and one output, two TSK type fuzzy IF-THEN rules are considered.

Rule 1: If $x$ is $A_{1}$ and $y$ is $B_{1}$, then $f_{1}=p_{1} x+q_{1} y+r_{1}$

Rule 2: If $x$ is $A_{2}$ and $y$ is $B_{2}$, then $f_{2}=p_{2} x+q_{2} y+r_{2}$

Where $x$ and $y$ are input variables, $f_{i}$ is the output and $A_{i}$ and $B_{i}$ represent the fuzzy sets which are defined over the input domain, and $p_{i}, q_{i}$ and $r_{i}$ are the parameters of linear polynomial in the $4^{\text {th }}$ layer of ANFIS network. The function of the nodes in all layers of ANFIS are listed in Table 1.

In the first layer, input values are converted to their corresponding subspaces by deploying bell-shaped membership functions in from of Equation 2. Selection of the shape and number of membership functions has a profound influence on the complexity and accuracy of ANFIS-based models. Therefore, bell-shaped membership functions, considering their smooth representation of input space are selected. The tuneable parameter sets $\left(a_{i}, b_{i}\right.$ and $\left.c_{i}\right)$ of these membership functions are determining the shape of membership function and are referred to as premise parameters. In the node function of layer one (see Table 1), $x$ and $y$ are the inputs to the node $i, A$ and $B$ are the linguistic labels, and $\mu(x)$ and $\mu(y)$ are bellshaped membership functions ranging from 0 to 1 .

$$
\mu(x)=\frac{1}{1+\left(\frac{x-c_{i}}{a_{i}}\right)^{2 b_{i}}}
$$


In the second layer, input signals from previous layer are multiplied and firing strengths of the fuzzy rules are obtained. Subsequently, in third layer, the firing strengths are normalised against each other. Then, in the fourth layer, the normalise d firing strengths are multiplied with the consequent parameters $\left(p_{i}, q_{i}\right.$ and $\left.r_{i}\right)$. Finally, in the fifth layer the final crisp output is achieved by summation of all incoming signals.

Table 1: Node functions of ANFIS layers.

\begin{tabular}{clcc}
\hline Layer & Node Function & Node Type & Tuneable Parameters \\
\hline $\mathbf{1}$ & $O_{1, i}=\mu_{A_{i}}(x)$ for $i=1,2$, or & Adaptive & $a_{i}, b_{i}$ and $c_{i}$ \\
& $O_{1, i}=\mu_{B_{i-2}}(y)$ for $i=3,4$ & & \\
\hline $\mathbf{2}$ & $O_{2, i}=w_{i}=\mu_{A_{i}}(x) \mu_{B_{i}}(y), \quad i=1,2$ & Fixed & - \\
\hline $\mathbf{3}$ & $\bar{w}_{i}=\frac{w_{i}}{w_{1}+w_{2}}, i=1,2$ & Fixed & $p_{i}, q_{i}$ and $r_{i}$ \\
\hline $\mathbf{4}$ & $O_{4, i}=\bar{w}_{i} f_{i}=\bar{w}_{i}\left(p_{i} x+q_{i} y+r_{i}\right)$ & Adaptive & - \\
\hline & & & \\
& $O_{5,1}=\sum_{i} \bar{w}_{i} f_{i}=\frac{\sum_{i} f_{i}}{\sum_{i} w_{i}}$ & & \\
\hline
\end{tabular}

\section{ANFIS Learning Algorithm}

As previously mentioned, the nodes in the first and fourth layers of ANFIS are adaptive and ANFIS model can be trained by tuning the parameters in these layers. The tuneable parameters in the first layer are $a_{i}, b_{i}$ and $c_{i}$, whereas the $p_{i}, q_{i}$ and $r_{i}$ for the fourth layer. ANFIS adopts a hybrid method to tune these parameters. This hybrid method has a forward and a backward pass as shown in Table 2.

Table 2: Hybrid learning algorithm of ANFIS.

\begin{tabular}{lll}
\hline Parameter & Forward Pass & Backward Pass \\
\hline Antecedent Parameters & Fixed & Gradient Decent \\
Consequent Parameters & Least Square Estimate & Fixed \\
\hline Signals & Node Outputs & Error Signals \\
\hline
\end{tabular}

In the forward pass the antecedent parameters in the first layer are fixed and the input signal propagates forward till fourth layer of ANFIS and by using the least square estimate the error between the model outputs and training data is calculated. Afterwards in the backward pass, consequent parameters in the fourth layer are fixed and the error signal is propagated backward through the network architecture. Then, by using the gradient decent algorithm, the antecedent parameters of first layer are updated.

\section{Data Preparation for Training the ANFIS Model}

The accuracy of model in the neural network-based techniques is highly dependent on the training data. Moreover, for an accurate model with a high prediction power, the excitation signal should cover the whole range of input domain. For a typical diesel ICE in heavy-duty applications the mass flow rate and temperature range of exhaust gases are 50-300 gm/s and 420-540 K, respectively, depending on the load condition and slope of the road. In this regard, a generic heat source (defined by Quoilin et. al. [10]) with 1470 data points in this range is chosen as the heat source of the evaporator. Furthermore, for 
the working fluid side of evaporator, the input temperature of evaporator is considered fixed and mass flow rate is changing in the range of 30-250 gm/s. Figs. 4 and 5 represent the excitation signals for training the ANFIS model. Three parameters of mass flow rate and temperature of the heat source and mass flow rate of refrigerant are applied as input data for exciting the FV model presented by Chowdhury et. al. [7]. By using this FV model, the evaporator is discretised to twenty control volumes to achieve the refrigerant outlet temperature and evaporator output power depicted in Fig. 6.

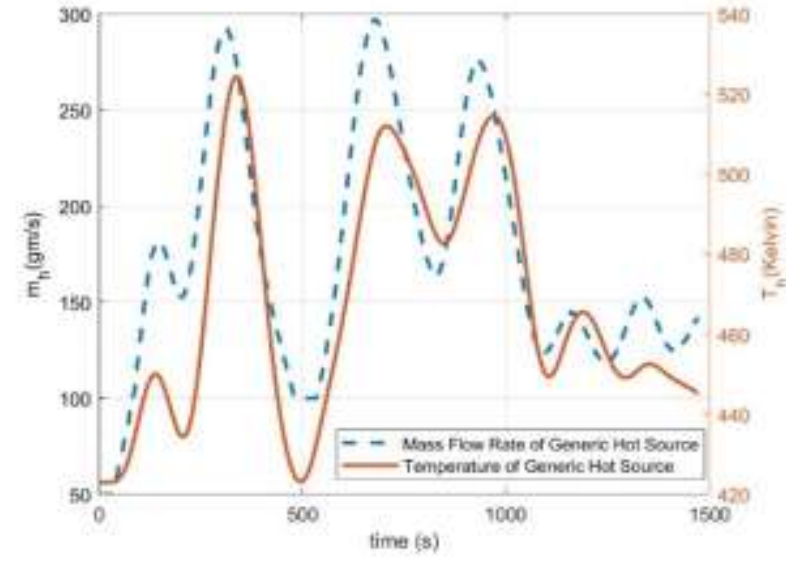

Fig. 4: Generic heat source specifications [10].

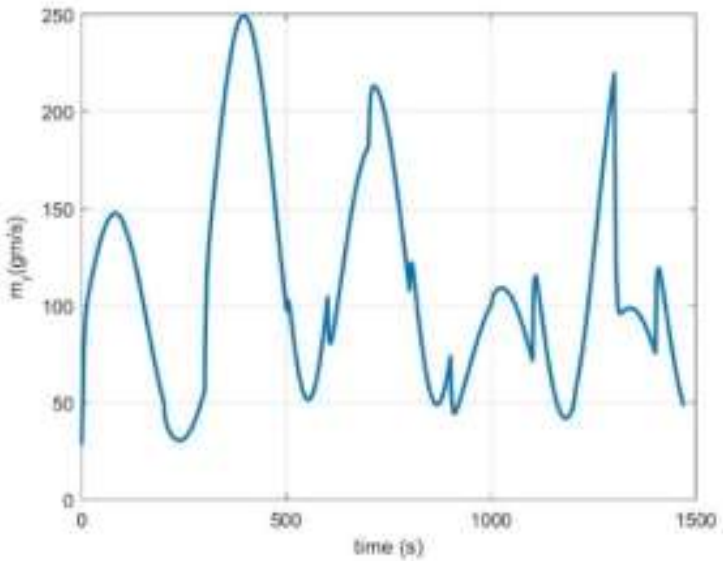

Fig. 5: Random profile refrigerant mass flow rate.

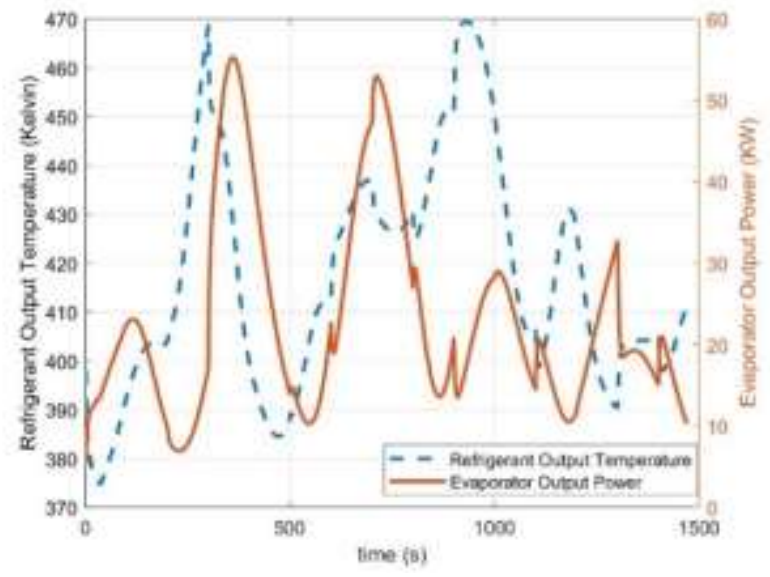

Fig. 6: Data obtained from FV model for Refrigerant output temperature $\left(T_{r}\right)$ and evaporator output power $\left(Q_{e v}\right)$.

\section{Results and Discussions}

After generating the input-output evaporator data from FV model of evaporator, this data was used to create the ANFIS model for the evaporator. It should be pointed out that the simulation time for the FV model was 3680 seconds. The K-means fuzzy clustering method is employed for classifying the data and generating the base Fuzzy Inference System (FIS) by setting the number and starting location of membership functions in the fuzzy sets. This base FIS is then trained using the ANFIS algorithm and the training data to achieve the ANFIS model of the evaporator. In the ANFIS algorithm the number of Epochs 
is set to 3000. For comparing the outputs of ANFIS model with FV model root mean square error (RMSE) represented in Equation 3 is used.

$$
R M S E=\sqrt{\frac{1}{n} \sum_{j=1}^{n}\left(\sigma_{j}-\overline{\sigma_{j}}\right)^{2}}
$$

In Equation 3, $n$ represents the total number of data points, $\sigma_{j}$ is the predicted ANFIS time series and $\bar{\sigma}_{j}$ is the time series calculated using the FV model. Furthermore, histograms of the error signals are drawn to indicate the mean and standard deviation of the error data.

Fig. 7 compares the response of ANFIS model and FV model to the training input data. As illustrated in Fig. 7(a) the RMSE for the refrigerant output temperature $\left(T_{r}\right)$ between the ANFIS and FV models is equal to 0.152. Moreover, from the histogram of error signal it is observable that the error mean and standard de viation are $5.44 \times 10^{-6}$ and 0.15 , respectively. Likewise, in Fig. 7(b) a comparison of ANFIS and FV models for the evaporator output power $\left(Q_{e v}\right)$ is made. The error mean is mostly around $-3.5 \times 10^{-7}$ with standard deviation of 0.035 , and the RMSE for the evaporator output power $\left(Q_{e v}\right)$ between two models is equal to 0.035 . The result of comparison between two models indicates the accuracy of ANFIS model for predicting the training data with an acceptable error margin. Furthermore, to evaluate the prediction power of the ANFIS model within the range of input signals, a new ramp profile for the mass flow rate of working fluid is chosen and used in both FV and ANFIS models, then a comparison between the two models is performed. As depicted in Fig. 8, the mass flow rate of working fluid for ramp profile is ranging from $30 \mathrm{lo} 250 \mathrm{gm} / \mathrm{s}$.
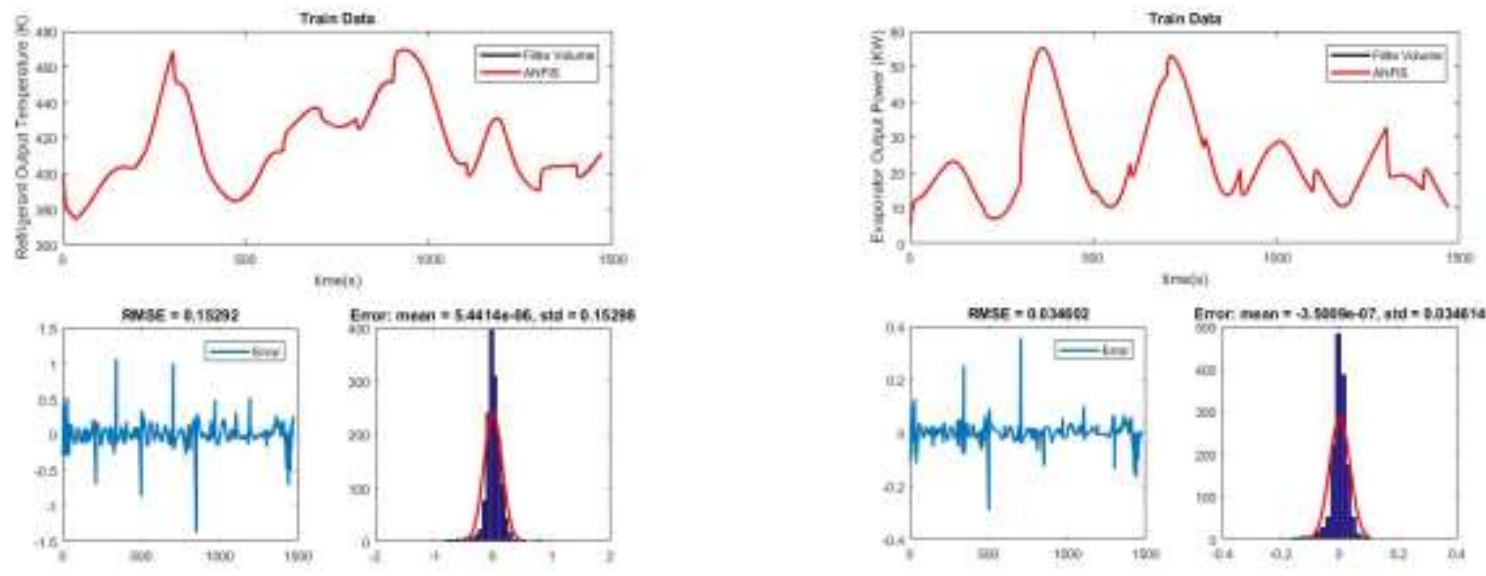

(a)

(b)

Fig. 7: Comparison of FV and ANFIS (Random profile): a) for refrigerant output temperature; b) for evaporator output power. 


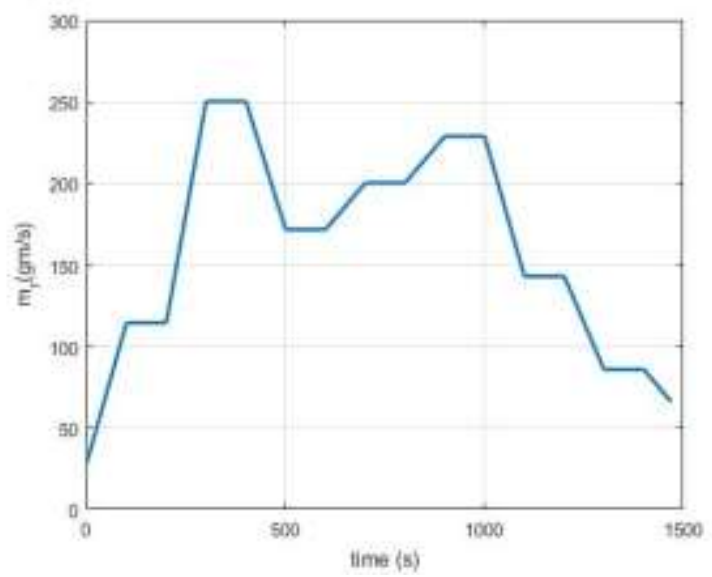

Fig. 8: Mass flow rate of refrigerant (ramp profile).

Fig. 9. Depicts the comparison of random profile input data for FV and ANFIS models. The obtained results (Fig. 9(a)) indicate the accuracy of ANFIS model in prediction of refrigerant output temperature $\left(T_{r}\right)$ achieved from FV model with RMSE, error mean and standard deviation of $1.33,-0.138$ and 1.32, respectively. Similarly, the ANFIS model can predict the evaporator outlet power $\left(Q_{e v}\right)$ attained from FV model with the test data set (Fig. 9(b)). The RMSE, error mean and standard deviation are $0.2,-0.082$ and 0.182 , respectively.
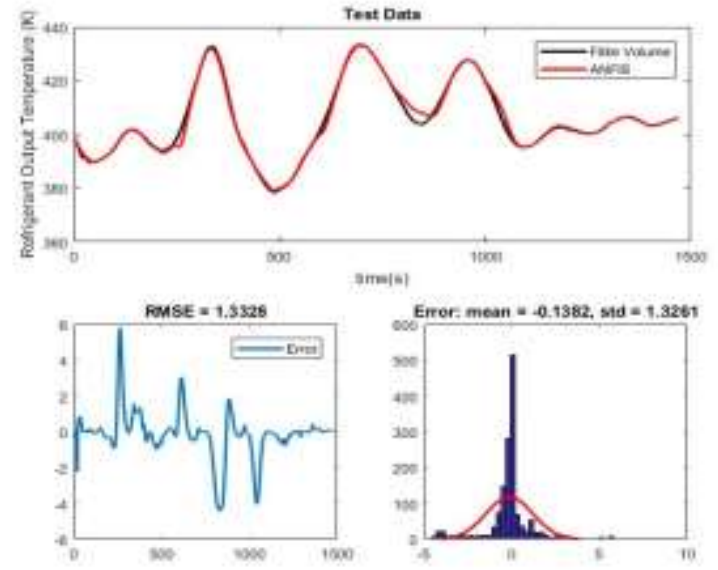

(a)
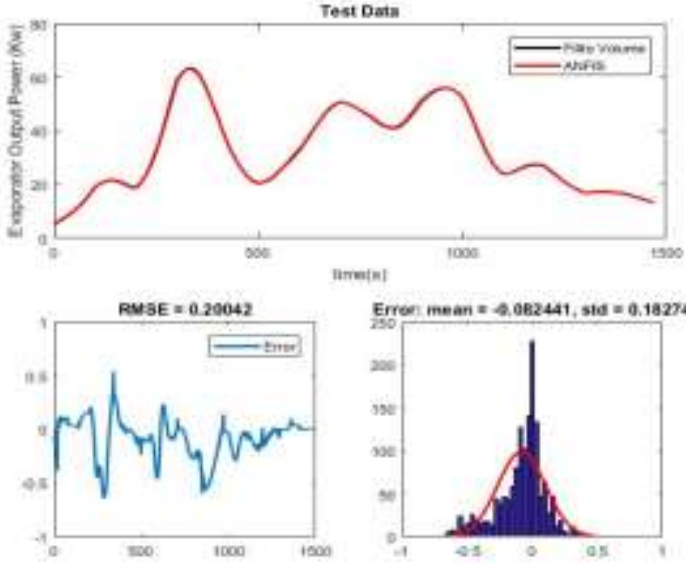

(b)

Fig. 9: Comparison of FV and ANFIS (Ramp profile): a) for refrigerant output temperature; b) for evaporator output power.

The comparison of obtained results from FV model and ANFIS model indicated an acceptable agreement between both model outputs. In addition, owing to the logic-based behaviour of ANFIS model the output results for 1470 data points are calculated in less than one second, which is significantly faster in comparison to 3680s for FV model.

\section{Conclusion}

In this study, an intelligent model of ANFIS was designed for modelling the evaporator nature. The ANFIS model was identified by input-output data achieved from FV model of the evaporator. Following are the main achievements of this study: 
- The time and effort of tuning the membership functions and rules in fuzzy sets are decreased substantially by deploying ANFIS method compared to the previous fuzzy method.

- The ANFIS model was compared with the FV model of evaporator by means of RMSE, error mean and standard deviation. Based on the obtained results for the training and test data, an acceptable agreement between the both model outputs observed.

- The ANFIS model substantially outperformed the FV model in term of simulation time. Consequently, it has a high potential to be considered for real-time applications.

\section{Acknowledgements}

The authors gratefully acknowledge the University of Sussex for the support in this research.

\section{References}

[1] R. El Chammas and D. Clodic, "Combined Cycle for Hybrid Vehicles," presented at the SAE 2005 World Congress \& Exhibition, Session : Advanced Hybrid Vehicle Powertrains (part 4 \& 5), Detroit, United States, 2005.

[2] R. Freymann, W. Strobl, and A. Obieglo, "The turbosteamer: A system introducing the principle of cogeneration in automotive applications," MTZ worldwide, vol. 69, no. 5, pp. 20-27, 2008.

[3] B. F. Tchanche, G. Lambrinos, A. Frangoudakis, and G. Papadakis, "Low-grade heat conversion into power using organic Rankine cycles - A review of various applications," Renewable and Sustainable Energy Reviews, vol. 15, no. 8, pp. 3963-3979, 2011.

[4] T. Wang, Y. Zhang, Z. Peng, and G. Shu, "A review of researches on thermal exhaust heat recovery with Rankine cycle," Renewable and Sustainable Energy Reviews, vol. 15, no. 6, pp. 2862-2871, 2011.

[5] S. Karellas, A. Schuster, and A. D. Leontaritis, "Influence of supercritical ORC parameters on plate heat exchanger design," Applied Thermal Engineering, vol. 33-34, no. 1, pp. 70-76, 2012.

[6] D. Wei, X. Lu, Z. Lu, and J. Gu, "Dynamic modeling and simulation of an Organic Rankine Cycle (ORC) system for waste heat recovery," Applied Thermal Engineering, vol. 28, no. 10, pp. 1216-1224, 2008.

[7] J. Chowdhury, B. Nguyen, and D. Thornhill, "Modelling of Evaporator in Waste Heat Recovery System using Finite Volume Method and Fuzzy Technique," Energies, vol. 8, no. 12, p. 12413, 2015.

[8] J. I. Chowdhury, P. Soulatiantork, and B. K. Nguyen, "Simulation of waste heat recovery system with fuzzy based evaporator model," in 2017 Asian Control Conference, ASCC 2017, 2018, vol. 2018-January, pp. 2143-2147.

[9] J. R. Jang, "ANFIS: adaptive-network-based fuzzy inference system," IEEE Transactions on Systems, Man, and Cybernetics, vol. 23, no. 3, pp. 665-685, 1993.

[10] S. Quoilin, R. Aumann, A. Grill, A. Schuster, V. Lemort, and H. Spliethoff, "Dynamic modeling and optimal control strategy of waste heat recovery Organic Rankine Cycles," Applied Energy, vol. 88, no. 6, pp. 2183-2190, 2011. 